\title{
$\mathbf{J}|\mathbf{A}| \mathbf{C} \mid \mathbf{S}$ \\ COMMUNICATIONS
}

Published on Web 02/26/2002

\section{Micelle-Induced Curvature in a Water-Insoluble HIV-1 Env Peptide Revealed by NMR Dipolar Coupling Measurement in Stretched Polyacrylamide Gel}

\author{
James J. Chou, ${ }^{\dagger}$ Joshua D. Kaufman, ${ }^{\ddagger}$ Stephen J. Stahl, ${ }^{\ddagger}$ Paul T. Wingfield, ${ }^{\ddagger}$ and Ad Bax ${ }^{\star}, \dagger$ \\ Laboratory of Chemical Physics, NIDDK, and Protein Expression Laboratory, NIAMDS, \\ National Institutes of Health, Bethesda, Maryland 20892
}

Received December 24, 2001

Integral membrane proteins are estimated to account for nearly one-quarter of all coding sequences in higher organisms, and more than half of all commercial drugs target this class of proteins. Despite the strong interest in such systems, study of their 3D structure remains limited by the inherent difficulty in growing 3D crystals suitable for X-ray diffraction and by their poor solubility for solution NMR studies. Solid-state NMR studies can be carried out on proteins anchored in phospholipid bilayers ${ }^{1}$ but frequently lack the degree of resolution observed in the liquid state. Alternatively, a variety of detergents ${ }^{2}$ is commonly used to study peptides and proteins that are known to interact with membranes or membrane proteins. The most popular NMR detergent is sodium dodecyl sulfate (SDS), although it is harsh and can have a denaturing effect. Short-chain phosphatidylcholines (PCs) such as dihexanoyl PC (DHPC) are gaining popularity as milder, zwitterionic detergents that also form small micelles with a mass of less than $20 \mathrm{kD} .{ }^{3,4}$ However, it has been argued that the strong curvature at the surface of such micelles provides a nonnatural environment. Small bicelles, which are self-assembling mixed micelles that consist of a mixture of short-chain and long-chain phospholipids, usually DHPC and dimyristoyl PC (DMPC), are presumed to be superior in this respect. ${ }^{5}$ However, no structural comparison of a peptide bound to micelles and bicelles is available to date. The small bicelles are disk-shaped, with DMPC making up the flat surface of the bilayered disk, and DHPC covering its rim. ${ }^{6}$ Most previous NMR studies using these small bicelles involved peptides that are somewhat water-soluble and which therefore are in a dynamic equilibrium between bicelle-bound and free in solution.

Here, we compare the structure of a peptide fragment of the HIV-1 envelope protein gp41 (residues 282-304) when bound to micelles and bicelles. The peptide is completely insoluble in water, and previous fluorescence and infrared experiments suggest it adopts an $\alpha$-helical structure that tightly associates with the lipid bilayer. ${ }^{7}$ Mutagenesis implicates its importance in viral infectivity, presumably by impacting an interaction between gp41 and the matrix protein. ${ }^{8}$ Comparison of the ${ }^{1} \mathrm{H}-{ }^{15} \mathrm{~N}$ HSQC correlation spectra at DMPC:DHPC molar ratios, $q$, of $0,0.1$, and 0.25 shows substantial changes in the NMR spectrum (Figure 1). At $q=0.5$, no further changes take place relative to $q=0.25$, but resonances broaden due to the slower tumbling of the larger bicelle (data not shown). Although not visible at the contour levels shown in Figure 1, the $q=0$ spectrum contains a large number of weak peaks in the vicinity of the main peaks, indicative of structural heterogeneity. In the $q=0.25$ bicelles, such minor components have vanishing intensity.

Although the HSQC spectra show chemical shift changes for different $q$ ratios, the chemical shift patterns remain preserved,

$\dagger$ Laboratory of Chemical Physics.

¥ Protein Expression Laboratory.

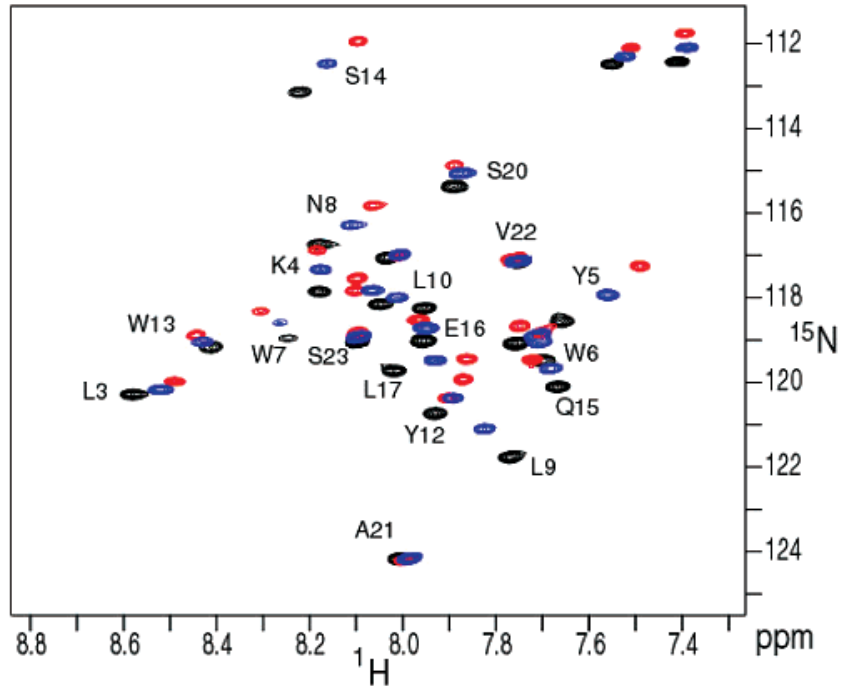

Figure 1. ${ }^{15} \mathrm{~N}-{ }^{-1} \mathrm{H}$ HSQC spectra (800 MHz) of $1 \mathrm{mM} \mathrm{gp} 41[282-304]$ in $300 \mathrm{mM}$ DHPC (red), in $q=0.1$ bicelles ( $350 \mathrm{mM}$ DHPC) (blue) and in $q=$ 0.25 bicelles (180 mM DHPC) (black). The gp41[282-304] peptide was expressed in Escherichia coli as a C-terminal extension to the SIV-gp41 ectodomain (residues $27-147$ ), and uniformly enriched with ${ }^{2} \mathrm{H},{ }^{13} \mathrm{C}$, and ${ }^{15} \mathrm{~N}$. The construct included an acid cleavage site (Asp-Pro) preceding the peptide, and the residue numbering used here starts from the N-terminal Pro.

indicating that the change in structure is relatively subtle. The conventional NMR approach to structure determination, based on NOEs and $J$ couplings, is generally not suitable for unambiguously identifying small structural changes. Instead, we therefore resort to dipolar couplings, which are exquisitely sensitive to bond vector orientation. Alignment of bicelles and detergent micelles in dilute liquid crystalline media ${ }^{9}$ is not possible because of the incompatibility of these media with detergent. In principle, a paramagnetic tag could be used to align the micelle/bicelle, ${ }^{10}$ but instead we utilize a recently described modification ${ }^{11}$ of the strain-induced alignment in a gel (SAG) method. ${ }^{12}$ In this method, the micelles/bicelles are soaked into a cylindrically shaped $6 \%$ polyacrylamide gel (acrylamide/bisacrylamide molar ratio of 80), initially $6 \mathrm{~mm} \times 9 \mathrm{~mm}$, which is subsequently radially compressed to fit within an NMR tube (i.d. $4.2 \mathrm{~mm}$ ), thereby increasing its length to $18 \mathrm{~mm}$.

Three different types of dipolar couplings are measured: ${ }^{1} D_{\mathrm{HN}}$, ${ }^{1} D_{\mathrm{C \alpha} C^{\prime}}$, and ${ }^{1} D_{\mathrm{C}^{\prime} \mathrm{N}}$. Values are listed as Supporting Information. In DHPC micelles, alignment is found to be stronger than in low- $q$ bicelles, indicating that the peptide-micelle deviates considerably from spherical symmetry. However, considering that phospholipid micelles and bicelles are soft and easily deformable particles, the correlation between their shape and alignment is expected to be less straightforward than for steric alignment of globular proteins. ${ }^{13}$ 

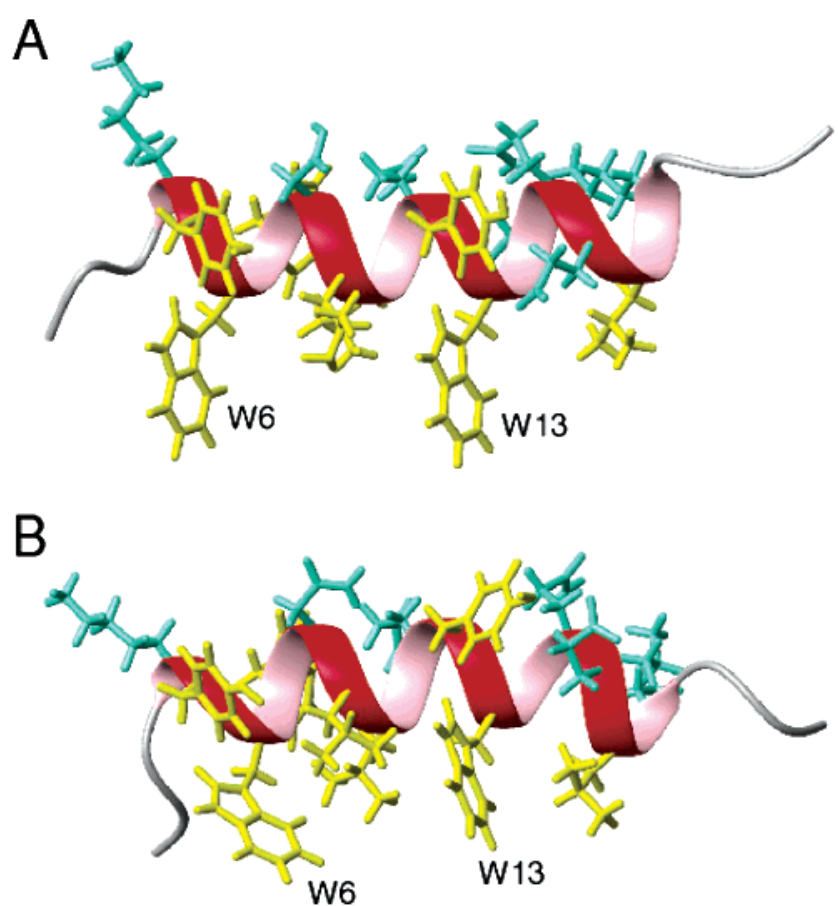

Figure 2. Structures of gp41[282-304] in (A) $q=0.25$ bicelles and (B) DHPC micelles, determined from dipolar couplings. Side chain $\chi_{1}$ angles are derived from ${ }^{3} J_{\mathrm{NC} \gamma}$ and ${ }^{3} J_{\mathrm{C}^{\prime}} \mathrm{C}$ couplings (Supporting Information). Hydrophobic and hydrophilic side chains are shown in yellow and aqua, respectively. The figure is made with the program MOLMOL. ${ }^{17}$

Only at higher $q$ values $(\geq 0.5)$ does the bicelle alignment rapidly increase (data not shown).

Using the program TALOS, $,{ }^{14} \mathrm{C}^{\alpha},{ }^{13} \mathrm{C}^{\beta}$, and ${ }^{13} \mathrm{C}^{\prime}$ chemical shifts (Supporting Information) indicate an $\alpha$-helical conformation in residues K4-K18. Similarly, a molecular fragment search ${ }^{15}$ through the PDB database only finds $\alpha$-helical fragments. Structure calculations were carried out using only dipolar restraints, $i$ to $i+4$ hydrogen bond restraints for $\mathrm{K} 4-\mathrm{K} 18$, and ${ }^{3} J_{\mathrm{NC} \gamma^{-}}$and ${ }^{3} J_{\mathrm{C}^{\prime} \mathrm{C} \gamma}$-derived $\chi_{1}$ torsion angles, starting from an initial structure with TALOSderived backbone angles. Force constants in the XPLOR protocol were adjusted such that the dipolar couplings in the final structure agreed to within the estimated measurement error (but not tighter) with the experimental data.

Figure 2 compares the structures of the peptide in DHPC micelles and in $q=0.25$ bicelles. A clear curvature is observed for the detergent micelle sample, with the hydrophobic residues on the inside. In contrast, a nearly straight helix, with a very slight curvature in the opposite direction, is seen for the bicelle sample. The helices are highly stable, with virtually no solvent exchange for the backbone amides over the entire helical region, but rapid solvent exchange near the $\mathrm{N}$ - and $\mathrm{C}$-termini. The $\alpha$-helix has a pronounced amphiphilic character, with Leu and Trp residues defining the hydrophobic surface. NOEs from the W6 and W13 indole $\mathrm{H}^{\mathrm{N}}$ to the DMPC alkyl methylene groups $(q=0.25)$ and to the DHPC alkyl $\mathrm{CH}_{3}(q=0)$ confirm that the Trp side chains are anchored deep into the hydrophobic lipid layer (Supporting Information). Ser and Tyr residues mark the "sides" of the helix, whereas the "outside" exposes Lys, Asn, Gln, and Gln residues to the aqueous solvent.

Evaluation of the backbone torsion angles indicates that in both micelles and bicelles the $\phi / \psi$ angles cluster around ideal $\alpha$-helical values. The tightness of the range of backbone angles observed in the bicelle phase for residues $4-18\left(\phi=-59 \pm 6^{\circ} ; \psi=-39 \pm 7^{\circ}\right)$ testifies to the regularity of this helix, whereas slightly more variation is found in the micelle-attached case $\left(\phi=-55 \pm 11^{\circ}\right.$; $\left.\psi=-43 \pm 12^{\circ}\right)$, presumably required for the helical bending.

Comparison of the two helices in Figure 2 strongly suggests that the curvature observed in the DHPC-bound form is induced by binding to the micelle, which in the absence of peptide would adopt a spherical shape with a radius, $r_{\text {micelle }}=18 \AA .{ }^{16}$ The radius of curvature observed for the peptide is considerably larger (35 \pm 5 $\AA$ ), indicative of an inherent degree of stiffness in the $\alpha$-helix. Presumably, $\alpha$-helices much longer than the one studied here would require more curvature, or stronger distortion of the DHPC micelle. Curvature of the bicelle-attached peptide $(r=70 \pm 10 \AA)$ is much weaker and in the opposite direction (Figure 2A), and probably results from the attractive interaction between the hydrophobic peptide residues and the phospholipid bilayer, whereas the charged $\mathrm{N}$ - and $\mathrm{C}$-termini are attracted to the aqueous phase. Interestingly, decreased ${ }^{15} \mathrm{~N}$ transverse relaxation rates indicate that the $\mathrm{N}$ - and C-terminal residues are subject to substantial internal motions, which are of roughly comparable magnitudes. Similarly, dipolar couplings near the ends of the peptide have considerably reduced but nonzero values, indicating that residual structure remains. However, the limited number of accessible dipolar couplings is insufficient to probe the average structure of these partially disordered termini.

We show that detergent micelles can induce significant strain in attached helical peptides and confirm that small bicelles are well suited for studying the detailed peptide structure in a biologically more relevant environment. Unambiguous identification of the weak curvature observed in the detergent micelle environment is generally not possible by conventional NMR, using NOEs and $J$ couplings, but is determined quantitatively by residual dipolar couplings.

Acknowledgment. This work is supported by the AIDS Targeted Anti-Viral Program of the Office of the Director of the NIH. J.J.C. is a GlaxoSmithKline Fellow of the Life Sciences Research Foundation.

Supporting Information Available: Tables with chemical shifts, dipolar couplings, ${ }^{3} J_{\mathrm{NC} \gamma}$ and ${ }^{3} J_{\mathrm{C}^{\prime} \mathrm{C} \gamma}$ couplings, and four strip plots taken through the ${ }^{15} \mathrm{~N}$-separated 3D NOESY spectra (PDF). This material is available free of charge via the Internet at http://pubs.acs.org.

\section{References}

(1) Marassi, F. M.; Opella, S. J. Curr. Opin. Struct. Biol. 1998, 8, 640-648; Fu, R. Q.; Cross, T. A. Annu. Rev. Biophys. Biomolec. Struct. 1999, 28 $235-268$

(2) Vinogradova, O.; Sonnichsen, F.; Sanders, C. R. J. Biomol. NMR 1998 11, 381-386; Damberg, P.; Jarvet, J.; Graslund, A. Methods Enzymol. 2001, 339, 271-285.

(3) Kessi, J.; Poiree, J. C.; Wehrli, E.; Bachofen, R.; Semenza, G.; Hauser, H. Biochemistry 1994, 33, 10825-10836.

(4) Arora, A.; Tamm, L. K. Curr. Opin. Struct. Biol. 2001, 11, 540-547.

(5) Vold, R. R.; Prosser, R. S.; Deese, A. J. J. Biomol. NMR 1997, 9, $329-$ 335; Sanders, C. R.; Prosser, R. S. Struct. Fold. Des. 1998, 6, 1227-1234.

(6) Luchette, P. A.; Vetman, T. N.; Prosser, R. S.; Hancock, R. E. W.; Nieh, M. P.; Glinka, C. J.; Krueger, S.; Katsaras, J. Biochim. Biophys. ActaBiomembr. 2001, 1513, 83-94; Glover, K. J.; Whiles, J. A.; Wu, G. H.; Yu, N. J.; Deems, R.; Struppe, J. O.; Stark, R. E.; Komives, E. A.; Vold, R. R. Biophys. J. 2001, 81, 2163-2171.

(7) Kliger, Y.; Shai, Y. Biochemistry 1997, 36, 5157-5169.

(8) Murakami, T.; Freed, E. O. J. Virol. 2000, 74, 3548-3554.

(9) Tjandra, N.; Bax, A. Science 1997, 278, 1111-1114.

(10) Ma, C.; Opella, S. J. J. Magn. Reson. 2000, 146, 381-384.

(11) Chou, J. J.; Gaemers, S.; Howder, B.; Louis, J. M.; Bax, A. J. Biomol. NMR 2001, 21, 377-382.

(12) Tycko, R.; Blanco, F. J.; Ishii, Y. J. Am. Chem. Soc. 2000, 122, 93409341; Sass, H. J.; Musco, G.; Stahl, S. J.; Wingfield, P. T.; Grzesiek, S. J. Biomol. NMR 2000, 18, 303-309.

(13) Zweckstetter, M.; Bax, A. J. Am. Chem. Soc. 2000, 122, 3791-3792

(14) Cornilescu, G.; Delaglio, F.; Bax, A. J. Biomol. NMR 1999, 13, 289-302.

(15) Delaglio, F.; Kontaxis, G.; Bax, A. J. Am. Chem. Soc. 2000, 122, $2142-$ 2143 .

(16) Tausk, R. J. M.; vanEsch, J.; Karmiggelt, J.; Voordouw, G.; Overbeek, J. T. G. Biophys. Chem. 1974, 1, 184-203.

(17) Koradi, R.; Billeter, M.; Wuthrich, K. J. Mol. Graph. 1996, 14, 51-55. JA017875D 